\title{
Sincronía en la comunicación pedagógica y heterogeneidad de los grupos en el nivel de conocimientos previos: efectos en el aprendizaje en aulas digitales
}

\author{
Luis Facundo Maldonado Granados* \\ Linda Alejandra Leal Urueña \\ Jaime Dario Castañeda \\ Milcon Montenegro Gamba \\ Carlos Lineros González \\ Jairo Pérez Rubio
}

Artículo recibido: 16-07-2007 y aprobado: 29-10-2008

\begin{abstract}
Comunication synchronicity and group heterogeneity, based on previous knowledge assessment: effects on learning in a digital classrooms
\end{abstract}

Resumen: Este artículo presenta los resultados de una investigación interesada en evaluar los efectos de la heterogeneidad de los grupos de estudiantes en el nivel de conocimientos previos y la sincronía en la comunicación de los mismos, sobre el aprendizaje de un dominio de conocimiento en un escenario de aula virtual. Se tomaron estudiantes de un curso de lógica matemática y se distribuyeron en dos grupos: uno integrado por estudiantes con conocimientos previos similares, y otro por estudiantes con conocimientos previos heterogéneos, los dos evaluados mediante un pre-test. Cada estudiante fue asignado aleatoriamente a uno de dos subgrupos: uno que usó como mecanismo de comunicación pedagógica el foro y otro que utilizó el chat. Por último, se diseñó un aula virtual siguiendo un Modelo de Reducción de Diferencias. La investigación concluye mostrando el efecto positivo del aprendizaje en contextos heterogéneos y la importancia del trabajo en equipo de los estudiantes. Se prueba que el Modelo de Reducción de Diferencias es un derrotero eficaz para lograr educación de alta calidad en aulas virtuales.

Proyecto cofinanciado por COLCIENCIAS y UNAD según contrato 192/05.

Palabras clave: Sincronía, chat, foro, aprendizajes previos, modelo de reducción de diferencias, aprendizaje autónomo, aula virtual, consolidación de grupo, liderazgo comunicativo.
Abstract: This article presents the results of a research addressed to test the effects of group heterogeneity, based on previous knowledge assessment, and the communication synchronicity on learning achievement of a knowledge domain in a virtual scenario. Students from a logic mathematics course were distributed into two groups: one integrated by students with similar previous knowledge, and other one with heterogeneous previous knowledge, assessed by a pretest. Each student was randomly assigned to one of the following subgroups: the first one, using as a means of communication a forum, and the second one using a chat. A virtual room was implemented following a Differences Reduction Model. This research shows a positive effect of heterogeneity as well as the collaboration of students on learning achievement, and it proves that a Differences Reduction Model is an effective way of accomplishing high quality education in virtual scenarios.

Key words: Synchronicity, chat, forum, previous knowledge, Difference Reduction Model, autonomous learning, virtual classroom, group consolidation, communication leadership.

\footnotetext{
* Investigadores Universidad Nacional Abierta y a Distancia, UNAD. lindalealau@gmail.com.
} 


\section{Planteamiento del problema}

Tomando un aula virtual como escenario de comunicación pedagógica y de aprendizaje, nos preguntamos si la organización homogénea o heterogénea de grupos, en el nivel de conocimientos previos, incide en el aprendizaje de un área de conocimiento.

Entendiendo el fenómeno del aprendizaje en términos de solución de problemas, construcción significativa de conceptos y competencias en un área o dominio de conocimiento, nos interesa verificar la incidencia de las interacciones sincrónicas y asincrónicas en el aprendizaje significativo.
Hablamos de sincronía cuando tenemos al menos dos procesos que, en un intervalo de tiempo, establecen entre sí relaciones de complementariedad e interacción (Sowa, 2000). Habrá mayor sincronía entre menor sea el tiempo para establecer el estado de acople entre los procesos. En la comunicación pedagógica, la sincronía se entiende como un conjunto de procesos de regulación mutua generados por agentes que se comunican y que, en tiempos relativamente cortos, entran en interacción.

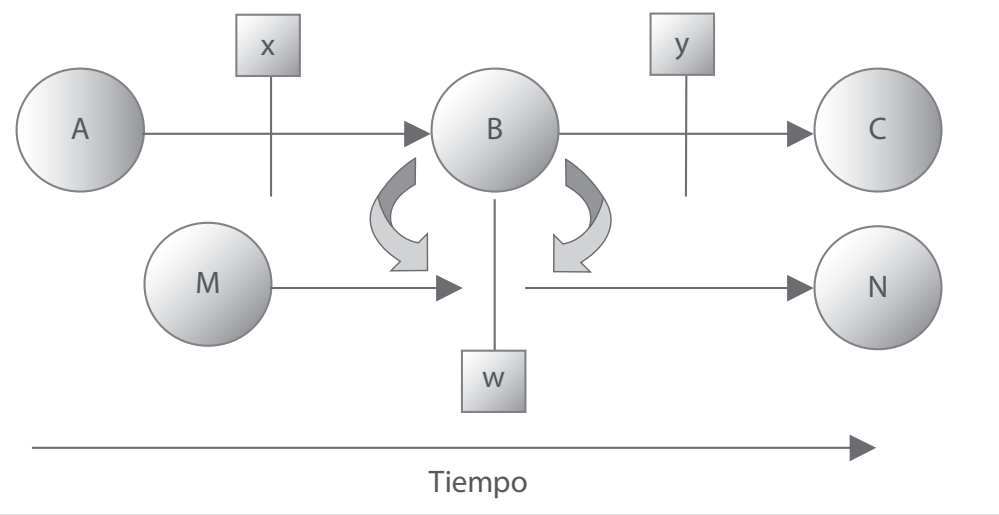

Ilustración 1. Representación en diagramas de Petri de la sincronización de dos procesos. El proceso de comunicación pedagógica involucra un estado $(A)$ en donde el conocimiento se encuentra representado o codificado junto a un evento emisión $(\mathrm{X}$ ), los cuales hacen que el docente pase a estado de espera de respuesta (B); el estado de espera (M) del estudiante con el evento recepción del estudiante (w) hacen que el proceso del docente incida en el proceso del estudiante y que este último pase al estado siguiente (N): información decodificada. La integración de los dos procesos requiere que éstos sean ajustados dentro de ciertos márgenes de tiempo. En la medida en que la integración se pueda hacer en intervalos más amplios en la línea del tiempo, los procesos serán más asincrónicos. Los procesos totalmente asincrónicos no se integran, a lo sumo pueden considerarse como procesos paralelos. 
La construcción de una pedagogía en aulas virtuales lleva a un análisis profundo de los mecanismos de comunicación pedagógica, que en estos escenarios tienen una capacidad más limitada que en los entornos convencionales. En un chat, por ejemplo, la emisión, lectura y respuesta de los mensajes están afectadas por la velocidad de transmisión, el número de participantes, la velocidad de escritura y la capacidad de los individuos de seguir una argumentación en busca de un objetivo. El foro, comparado con el chat, es un mecanismo de comunicación que impone menos demandas de velocidad y atención por parte del usuario, ya que acepta un tiempo mayor entre la emisión del mensaje y la recepción de la respuesta. El chat, al exigir menos tiempo de espera para la integración de los procesos de emisión y recepción de la comunicación pedagógica, es más sincrónico que el foro, pero, complementar el ciclo de la comunicación hace necesario garantizar siempre la integración entre los dos procesos, de tal manera que no se presente la asincronía total.

Aparentemente el foro procesaría mensajes a una velocidad menor, favoreciendo un tratamiento más cuidadoso de la discusión y posiblemente sea el medio más pertinente para la solución de problemas complejos; mientras que el chat, hipotéticamente, puede considerarse más apropiado para la consolidación de grupos debido a la velocidad de la comunicación.

El intercambio entre pares adquiere mayor relevancia en estos entornos, se hace más visible, más frecuente, y las relaciones del docente con el grupo toman rasgos distintos. Los cursos y actividades pedagógicas en aulas virtuales se rigen por esquemas controlados por docentes o tutores que orientan la comunicación pedagógica, en este sentido, el proyecto considera para su aplicación el esquema denominado Reducción de Diferencias (Dulonsky y Hertzog, 1998; Thiede, Anderson y Therrialt, 2003).

Maldonado (2005), desde la perspectiva del Modelo de Reducción de Diferencias (MRD) considera que las informaciones, provenientes de la meta y del estado actual del aprendizaje, convergen en un modelo mental o juicio de meta-memoria que determina lo que falta para que el estado actual sea el estado ideal y decide qué acción tomar para generar nuevos cambios en la actualidad, de manera que se puedan reducir sus diferencias (ver ilustración 2).

La información sobre el estado actual del aprendizaje depende de dos fuentes de información: sobre la meta y sobre las soluciones que un sujeto es capaz de ejecutar frente a una clase de problema que está aprendiendo a resolver. En el juicio de meta-memoria se sintetizan las dos fuentes de información, pero también es necesario considerar que el sujeto requiere conocer qué acciones puede tomar para modificar: a) la comprensión de la meta, b) la información sobre su aprendizaje actual, y c) su capacidad de resolver el problema.

La comparación entre estado actual y estado meta es fundamental en el diseño de entornos para el aprendizaje autodirigido. El reto consiste en adecuar mecanismos de acceso a la información sobre el estado actual del aprendizaje e incentivar la comparación con el estado meta.

Por otra parte, es importante la disponibilidad de estrategias de aprendizaje en memoria de largo plazo o en memoria externa para que se pueda 


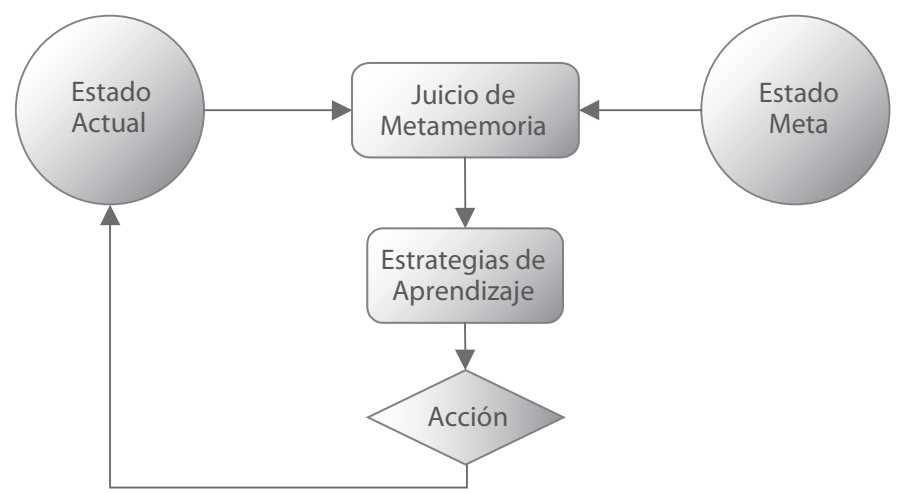

llustración 2. Modelo de Reducción de Diferencias (MRD).

El juicio de meta-memoria compara el estado actual del aprendizaje con el estado meta y activa la selección de estrategias de la librería actual, en memoria de largo plazo o en memoria externa, y genera la decisión de ejecutar una acción orientada a mover el estado actual del aprendizaje en dirección a la meta.

activar la iniciativa del estudiante. El ambiente de aprendizaje puede disponer de librerías, de estrategias que pueden ser consultadas por los usuarios.

El proceso de Reducción de diferencias puede verse favorecido por la interacción entre pares que comparten estados de conocimientos previos equiparables. Algunas investigaciones sobre aprendizaje colaborativo, establecen que el trabajo entre grupos homogéneos favorece la simetría en la comunicación y con ello, se aumenta el control de los procesos de aprendizaje. Por otra parte, es necesario resaltar que en los grupos heterogéneos se presenta una comunicación asimétrica (Dillenbourg, Mendelsohn y Schneider, 1994), que permite esperar que el afianzamiento de los grupos de estudiantes favorezca el trabajo en sociedades y finalmente, afecte el aprendizaje. Este proceso debería dar como resultado una mayor eficiencia y eficacia en la comunicación y una mejora en cuanto a las competencias desarrolladas por los estudiantes.
Bodzin (2000) investigó el foro virtual como escenario de comunicación entre estudiantes de educación que desarrollaban práctica docente y encontró que esta forma asincrónica de comunicación dio lugar al fortalecimiento de los vínculos entre los estudiantes, con lo cual se logró formar una "comunidad virtual de aprendizaje”. En este escenario se superaron las actitudes iniciales negativas acerca del uso de la web como instrumento de aprendizaje, se favoreció el pensamiento reflexivo, se superó la sensación de aislamiento que frecuentemente sentían los practicantes y se generó una dinámica de trabajo en la cual se exponían situaciones particulares de la práctica y se discutían soluciones a problemas.

Harpe Mackenzie y Technikon (2002) hacen un estudio exploratorio de las intervenciones de las personas en el chat, categorizándolas como negativas o positivas, el estudio señala la necesidad de lograr una comprensión profunda de las dinámicas que se dan en un escenario como éste, de manera que se pueda 
determinar su uso pedagógico. Se plantea además que el chat es un escenario reciente de investigación.

Desde esta perspectiva, la investigación sobre la relación entre las variedades de sincronía en la comunicación, se muestra el desarrollo de competencias comunicativas y el aprendizaje significativo como un área de investigación todavía incipiente. Sin embargo, el uso cada vez más extendido de aulas virtuales en programas educativos muestra la relevancia social de su estudio.

\section{Diseño metodológico}

Para evaluar el efecto de la sincronía en este proceso se distribuyeron en dos grupos los estudiantes de un curso de lógica matemática, teniendo en cuenta como criterio para la selección el nivel de conocimiento previo de los estudiantes sobre la asignatura. El primer grupo estuvo integrado por estudiantes con conocimientos previos similares, y el otro por estudiantes con niveles de conocimientos previos heterogéneos. Para efectos de la selección se diseñó y aplicó una prueba de entrada.

La muestra estuvo conformada por 80 estudiantes de primer semestre de las cuatro facultades de la Universidad Nacional Abierta y a Distancia (UNAD), se realizó una selección aleatoria a partir del listado de los estudiantes inscritos al curso mencionado.

La selección del grupo heterogéneo se realizó de la siguiente manera: se ordenaron de menor a mayor los puntajes obtenidos por los estudiantes en la prueba de entrada, luego se seleccionaron los primeros 20 puntajes y los últimos 20. El grupo homogéneo estuvo constituido por los demás estudiantes de la muestra.
El grupo homogéneo y el grupo heterogéneo, cada uno por separado, se distribuyó aleatoriamente en dos subgrupos de tal manera que 20 estudiantes participaran en el chat y 20 en el foro. Esta distribución dio lugar a un arreglo matricial 2x2 que se presenta en la Tabla 1.

\begin{tabular}{lcc}
\hline Grupos / Mecanismo & Foro & Chat \\
\hline Homogéneo & G1 (20) & G2 (20) \\
\hline Heterogéneo & G3 (20) & G4 (20) \\
\hline
\end{tabular}

Tabla 1. Distribución de los cuatro subgrupos en las condiciones experimentales.

Para el desarrollo de cada capítulo del curso, los grupos que interactuaron de manera asincrónica tuvieron dos sesiones de foro semanales: una entre el domingo y el miércoles y otra entre el miércoles y el sábado.

Los grupos que se comunicaron sincrónicamente a través del chat tuvieron dos sesiones semanales, distribuidas así: una el lunes y otra el jueves. Los grupos del chat estaban conformados por 10 estudiantes y un tutor.

\section{Descripción del aula virtual}

La adaptación del aula virtual al Modelo de Reducción de Diferencias (MRD), fue realizada siguiendo el modelo presentado en la ilustración 2. Para establecer la información sobre el estado actual del aprendizaje fueron diseñados dos componentes: el primero, denominado “¿Hacia donde me dirijo?”, proporciona información de forma hipertextual sobre la meta de aprendizaje propuesta para cada unidad y el segundo, llamado “¿Cómo puedo lograrlo?”, incluye un conjunto de ejercitadores o problemas ya resueltos, que pueden ser usados por el estudiante, por una parte, para 
monitorear y obtener información sobre su desempeño y por otra, como apoyo a la solución de los problemas que le son propuestos para que aprenda sobre lógica matemática.

La información proveniente de estos dos componentes, meta y estado actual del aprendizaje, genera un modelo mental o juicio de meta-memoria en el estudiante, de manera que le permite identificar lo que le falta para que el estado actual sea el estado ideal.

La toma de decisiones, por parte del estudiante, acerca de qué tipo de acciones debe seguir para generar cambios en el estado actual y aproximarse a la meta, incluye la comprensión de la meta, la información sobre su aprendizaje actual y su capacidad de resolver el problema.

La comparación entre estado actual y estado meta en el aprendizaje es un aspecto fundamental en el diseño del aula virtual, puede darse cada vez que un estudiante resuelve un problema, pues el ejercitador evalúa la respuesta y presenta información sobre el resultado obtenido. En este sentido, es indispensable que exista disponibilidad de estrategias de aprendizaje en memoria de largo plazo o en memoria externa, que, para el presente estudio, se trabajó con el diseño de una librería de estrategias de aprendizaje para ser consultadas y aplicadas por los estudiantes.

\section{Estructura del curso virtual de lógica matemática}

El curso virtual de Lógica Matemática fue diseñado con el fin de apoyar el proceso de aprendizaje de los estudiantes de forma autónoma y colaborativa, es decir, que desde un trabajo autónomo, individual, se llegue a un trabajo en equipo y a la exposición de ideas que sean valoradas y desarrolladas por los demás. El curso está pensado en unidades, cada una de las cuales está estructurada en cinco secciones, a saber:

\section{¿Qué he aprendido?}

Corresponde a un conjunto de cuestionarios en línea que tienen el propósito de autoevaluar los conocimientos que se van desarrollando con el estudio de cada una de las unidades.

\section{¿Hacia dónde me dirijo?}

En esta sección se hace una presentación hipertextual de las metas de aprendizaje propuestas para cada unidad, su propósito es garantizar la comprensión de las metas de aprendizaje.

\section{¿Cómo puedo lograrlo?}

Corresponde a un conjunto de recursos de información entre los que se encuentran los módulos en los que se desarrollan los contenidos temáticos de cada unidad; escritos en forma hipertextual de manera que el estudiante pueda profundizar en cada tema, encuentre vínculos a lecturas adicionales disponibles en línea y un conjunto de ejercitadores que le permiten desarrollar habilidades para la solución de los problemas de lógica matemática.

\section{¿Con quiénes puedo contar?}

En esta sección se hace una programación semanal de las sesiones de chat o de foros con los temas y problemas propuestos para resolver de manera colaborativa.

\section{¿Sé lo que sé?}

Evaluación final de cada unidad del curso de lógica matemática. 


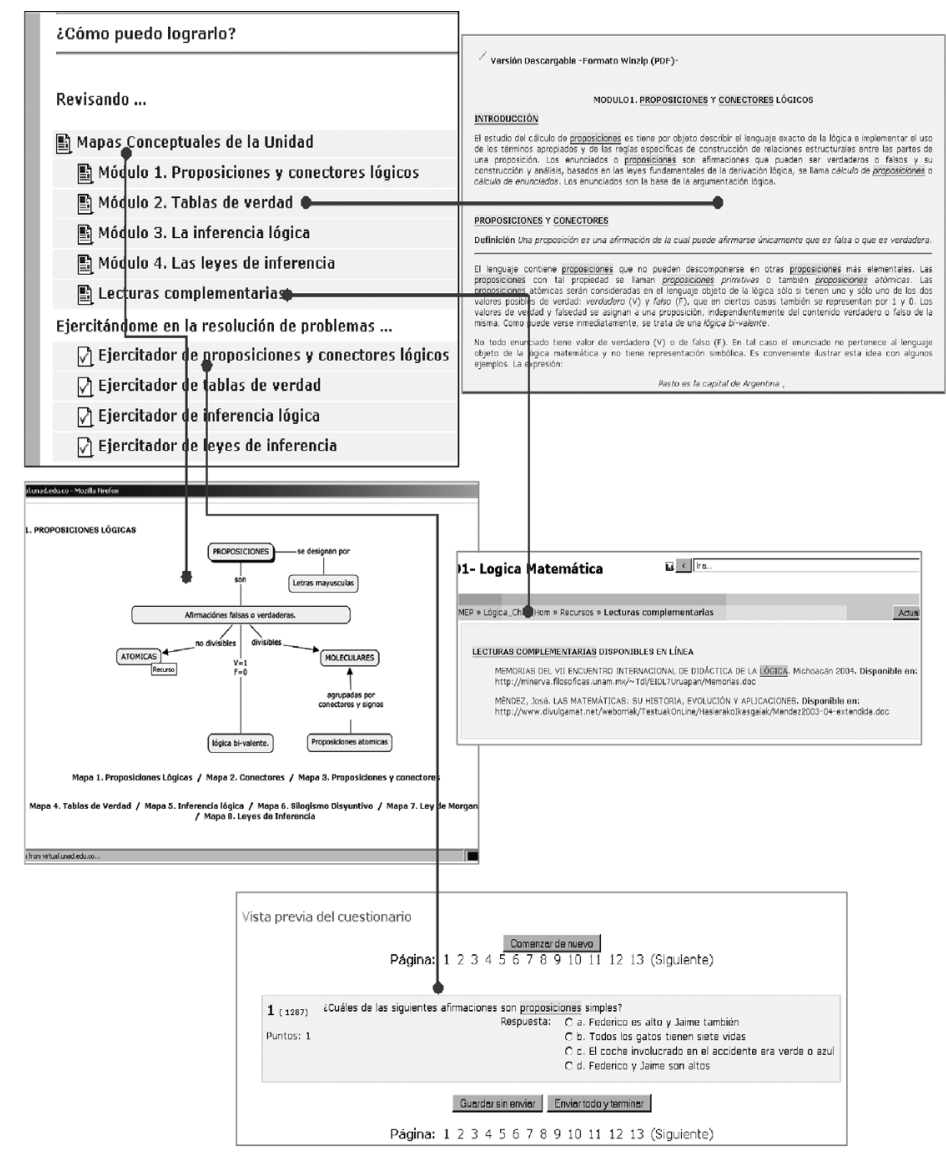

llustración 3. Recursos del componente ¿Cómo puedo lograrlo?

\section{Análisis de resultados}

\section{Primera Unidad - Lógica proposicional}

A la evaluación de la primera unidad se

Tabla 2. Se encontró una deserción de presentaron un total de 52 estudiantes $13 \%$ de los estudiantes inscritos inicialdistribuidos según se muestra en la mente en el curso.

\begin{tabular}{lccc}
\hline \multicolumn{1}{c}{ Grupo } & Promedio & Desviación estándar & Número de sujetos \\
\hline Chat homogéneo & 4.03 & 1.49 & 16 \\
\hline Chat heterogéneo & 4.35 & 1.66 & 13 \\
\hline Foro homogéneo & 2.93 & 0.89 & 10 \\
\hline Foro heterogéneo & 4.73 & 1.85 & 13 \\
\hline Todos los Grupos & 4.07 & 1.63 & 52 \\
\hline
\end{tabular}

Tabla 2. Medias del rendimiento en la primera unidad. 
El análisis factorial de varianza muestra diferencias significativas asociadas con la heterogeneidad como variable independiente, $(\mathrm{F}(1,48)=5.96 ; \mathrm{p}<0.02$, Tabla 3). Esto muestra que la media de los grupos heterogéneos (4.54) es significativamente más alta que la de los grupos homogéneos (3.48). Los pro- medios correspondientes a los grupos de chat (4.19) y de los grupos de foro (3.83) no son significativamente diferentes $(\mathrm{F}(1,48)=0.70 ; \mathrm{p}<0.40)$. El valor de la desviación estándar es menor para los grupos homogéneos, lo cual significa que éstos siguen tendiendo a resultados similares en cuanto aprendizaje.

\begin{tabular}{lcccccc}
\hline & Df Effect & MS Effect & Df Error & MS Error & F & p-level \\
\hline 1 Sincronía & 1 & 1.68 & 48 & 2.39 & 0.70 & 0.40 \\
\hline 2 Heterogeneidad & 1 & 14.27 & 48 & 2.39 & 5.96 & 0.02 \\
\hline 12 Sinc - Heterog & 1 & 7.00 & 48 & 2.39 & 2.92 & 0.09 \\
\hline
\end{tabular}

Tabla 3. Resumen de efectos del análisis factorial de varianza.

No se observa interacción significativa entre las variables homogeneidad $\mathrm{y}$ heterogeneidad $(\mathrm{F}(1,48)=2.92 ; \mathrm{p}<.09)$, ver ilustración 4 . Si bien los valores de F son mayores que la unidad, y la gráfica muestra que se cruzan las líneas que representan la sincronía y la heterogeneidad, el nivel de significación no llega a ser menor que 0.05 .

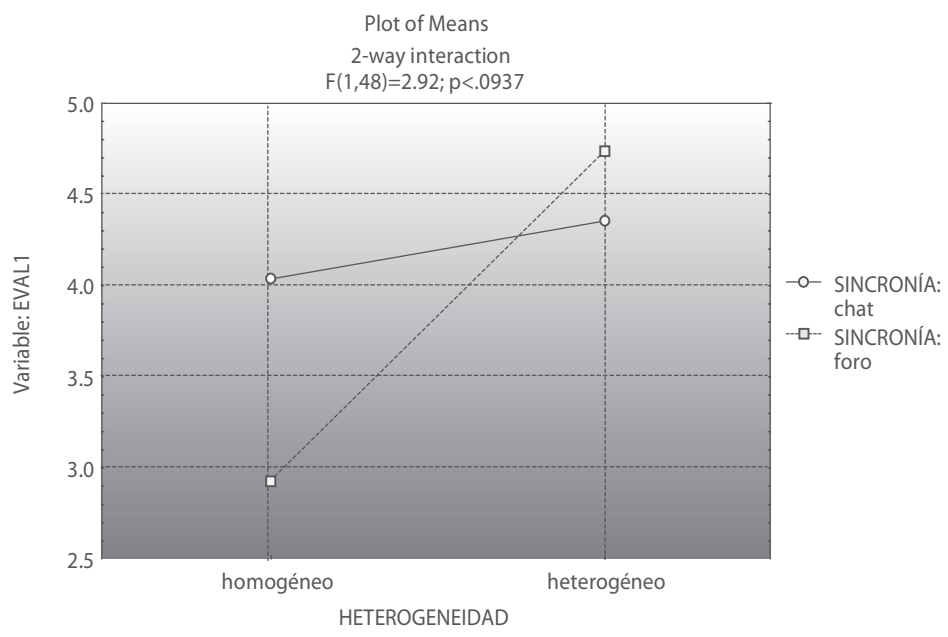

Ilustración 4. Interacción de variables primera evaluación.

\section{Segunda Unidad - Lógica de predicados}

A la evaluación de la segunda unidad se presentaron un total de 40 estudiantes distribuidos según se muestra en la Tabla 4.
El análisis factorial de varianza muestra diferencias significativas en la heterogeneidad como variable indepen- 
diente, $(\mathrm{F}(1,36)=4.43 ; \mathrm{p}<.042$, Tabla 4). Esto muestra que la media de los grupos heterogéneos (7.24) es significativamente más alta que la de los grupos homogéneos (6.03). El promedio de los grupos de chat (7.00) y el de los grupos de foro (6.27) no es significativamente diferente $(\mathrm{F}(1,36)=1.59 ; \mathrm{p}<0.2154)$. Se observa que con el paso del tiempo las desviaciones son más parecidas en todos los grupos.

\begin{tabular}{lccc}
\hline \multicolumn{1}{c}{ Grupo } & Promedio & Desviación estándar & Número de sujetos \\
\hline Chat homogéneo & 6.60 & 1.68 & 12 \\
\hline Chat heterogéneo & 7.41 & 1.88 & 9 \\
\hline Foro homogéneo & 5.47 & 1.79 & 7 \\
\hline Foro heterogéneo & 7.08 & 1.76 & 12 \\
\hline Todos los Grupos & 6.73 & 1.82 & 40 \\
\hline
\end{tabular}

Tabla 4. Medias del rendimiento en la segunda unidad.

\begin{tabular}{lcccccc}
\hline & Df Effect & MS Effect & Df Error & MS Error & F & p-level \\
\hline 1 Sincronía & 1 & 4.98 & 36 & 3.13 & 1.59 & 0.21 \\
\hline 2 Heterogeneidad & 1 & 13.88 & 36 & 3.13 & 4.43 & 0.04 \\
\hline 12 Sinc - Heterog & 1 & 1.51 & 36 & 3.13 & 0.48 & 0.49 \\
\hline
\end{tabular}

Tabla 5. Resumen de efectos del análisis factorial de varianza.

\section{Análisis integrado de las dos unidades}

El análisis factorial de varianza, introducido como tercera variable en la unidad $(\mathrm{F}(1,32)=30.49 ; \mathrm{p}<0.000004)$, muestra diferencias significativas entre esta variable y la de heterogeneidad $(\mathrm{F}(1,32)=8.48 ; \mathrm{p}<.006)$, ver Tabla 6 , mientras que no sucede lo mismo con la variable sincronía. Otro dato importante es que no se observa un efecto de interacción significativo entre las variables consideradas.

En resumen, puede decirse que se presentaron diferencias en el aprendiza- je de los estudiantes en cuanto a la lógica proposicional y la lógica de predicados, la primera con media global de 4.40 y la segunda de 6.76. De la misma manera, se observa que los grupos heterogéneos tienden a presentar mejores resultados que los grupos homogéneos en las dos unidades desarrolladas, por otro lado, las variables de uso del chat o del foro no cambian significativamente en la muestra de referencia, sea antes o después del desarrollo de las dos unidades del curso.

\begin{tabular}{lcccccc}
\hline & Df Effect & MS Effect & Df Error & MSError & F & p-level \\
\hline 1 Sincronía & 1 & 11.12 & 32 & 3.253269 & 3.41758 & .073764 \\
\hline 2 Heterogeneidad & 1 & 27.60 & 32 & 3.253269 & 8.48385 & .006483 \\
\hline 3 Unidad & 1 & 92.86111 & 32 & 3.045936 & 30.48689 & .000004 \\
\hline 12 & 1 & 5.33085 & 32 & 3.253269 & 1.63861 & .209718 \\
\hline 13 & 1 & .01249 & 32 & 3.045936 & .00410 & .949333 \\
\hline 23 & 1 & .00045 & 32 & 3.045936 & .00015 & .990381 \\
\hline 123 & 1 & .47043 & 32 & 3.045936 & .15444 & .696930 \\
\hline
\end{tabular}

Tabla 6. Resumen de efectos del análisis factorial de varianza. 


\section{Análisis de los ejercitadores}

Incluimos en el estudio el análisis del efecto de los ejercitadores en el aprendizaje, éste es el mecanismo diseñado en el sistema para activar la reducción de diferencias, de acuerdo con a lo planteado en el marco conceptual.

Se diseñaron ejercitadores en forma de bancos de preguntas sobre el contenido temático de las unidades y se pusieron a disposición permanente de los estudiantes. Para el análisis, se consideró en primer lugar, el resultado obtenido por el estudiante la última vez que utilizó el ejercitador y en segundo lugar, los resultados obtenidos por el mismo en la evaluación de la unidad, además, se usó el modelo de regresión múltiple con estas dos variables.

\section{Ejercitadores Primera Unidad}

Se dispusieron cuatro ejercitadores para esta unidad. La tabla 7 muestra los valores obtenidos para hacer el análisis de regresión utilizando los resultados de los estudiantes en los cuatro ejercitadores.

La $\mathrm{F}$ asociada con el modelo $\mathrm{F}(4,54)$ tiene un valor de 113,40, con un nivel de significación $\mathrm{p}<0,00000$, lo cual indica que el modelo que integra los cuatro ejercitadores explica los resultados de la primera evaluación. $\mathrm{La} \mathrm{R}^{2}=0,88573855$ muestra que el porcentaje de varianza explicada es del $88 \%$. El peso de Beta dentro de la ecuación es significativo para los ejercitadores uno y dos, seguido en su orden por el ejercitador cuatro y con menor efecto el ejercitador tres.

\begin{tabular}{llccccc}
\hline & BETA & St. Err.of BETA & B & St. Err.of B & t(54) & p-level \\
\hline EJERC 1 & 0,39 & 0,15 & 0,32 & 0,120874 & 2,67 & 0,009886 \\
\hline EJERC 2 & 0,27 & 0,13 & 0,20 & 0,101338 & 2,01 & 0,049520 \\
\hline EJERC 3 & 0,13 & 0,14 & 0,16 & 0,168714 & 0,93 & 0,358237 \\
\hline EJERC 4 & 0,19 & 0,17 & 0,28 & 0,203253 & 1,39 & 0,171075 \\
\hline
\end{tabular}

Tabla 7. Análisis de regresión ejercitadores primera unidad.

\section{Ejercitadores Segunda Unidad}

Se dispusieron dos ejercitadores para esta unidad. La tabla 8 muestra los resultados del análisis de regresión.

La $\mathrm{F}$ asociada con el modelo $\mathrm{F}(2,56)$ tiene un valor de 626,73, con un nivel de significación $\mathrm{p}<0,00000$, lo cual indica que el modelo que integra como predictores a los dos ejerc itadores, explica los resultados de la segunda evaluación. La $\mathrm{R}^{2}=0,95570694$ muestra que el porcen- taje de variable explicada es del 95\%. En su orden, los pesos de beta dentro de la ecuación muestran alta significación del efecto del ejercitador 5. El ejercitador 6 tiene un efecto notorio aunque no resulta significativo.

Los datos estadísticos muestran en consecuencia, un efecto significativo del uso de los ejercitadores sobre el aprendizaje evaluado en las dos unidades.

\begin{tabular}{ccccccc}
\hline & BETA & St. Err.of BETA & B & St. Err.of B & t(54) & p-level \\
\hline EJER 5 & .614703 & .197468 & .512025 & .164484 & 3.112923 & .002916 \\
\hline EJER 6 & .365941 & .197468 & .320446 & .172918 & 1.853166 & .069130 \\
\hline
\end{tabular}

Tabla 8. Análisis de regresión ejercitadores segunda unidad. 


\section{Interpretación de resultados}

Frente a nuestra pregunta inicial de si la organización homogénea o heterogénea de grupos, en el nivel de conocimientos previos, incide en el aprendizaje de un dominio de conocimiento en aula virtual, los datos estadísticos de esta investigación sugieren una respuesta positiva.

En contraposición a la interpretación de Dillenbourg (1994), según la cual la simetría entre grupos homogéneos favorecería la comunicación y regulación en los estudiantes, esta investigación muestra que la comunicación de grupos heterogéneos permite generar una mejor regulación de los procesos de aprendizaje hacia una meta formulada explícitamente en el aula virtual. De acuerdo con estos resultados, podemos postular el efecto significativo, de la comunicación entre los grupos heterogéneos, para el aprendizaje.

Posiblemente los estudiantes más avanzados generan procesos de comunicación efectiva sobre el dominio de conocimiento, con lo cual se logra que los estudiantes menos avanzados progresen consistentemente en el alcance de las metas de aprendizaje. Cuando los grupos son más homogéneos la percepción de metas y de estrategias de aprendizaje efectivas es más cercana, y en consecuencia, la dinámica de intercambio es menor que en los grupos heterogéneos. Probablemente en los grupos homogéneos se den menos desacuerdos para resolver el problema que en los grupos heterogéneos y en consecuencia la demanda para procesar información sea menor.

En cuanto a la pregunta inicial, sobre el efecto de las diferencias en la sincronía de la comunicación pedagógica para el aprendizaje en aulas virtuales, los datos estadísticos sugieren considerar que no hay diferencias entre grupos que usan el chat o el foro. Esto quiere decir que el aprendizaje no es sensible a las variaciones temporales de la comunicación cuando el límite de la variación de tiempo corresponde a cuatro días. Se hace necesario entonces, realizar investigaciones en las que las variaciones de tiempo sean mayores a las consideradas en este estudio.

La tercera conclusión está relacionada con la interacción entre las variables consideradas en el estudio. Según los resultados obtenidos, no se percibe efecto de interacción entre éstas, más aún, podría pensarse que en el largo plazo se mantienen los resultados, obtenidos en este estudio, de la comparación entre primera y segunda unidad, que presentan una disminución del efecto de la interacción sobre el aprendizaje. En términos prácticos, tanto en foro como en chat, los resultados de aprendizaje son mejores cuando los grupos son heterogéneos.

La comparación de los resultados de las unidades hace pensar en diferencias generadas por el dominio de conocimiento, pero también, en la influencia de factores estructurales del Modelo de Reducción de Diferencias, en particular sobre el uso de ejercitadores como mecanismos que habilitan el monitoreo, los juicios de metamemoria y el control autónomo de las actividades conducentes al aprendizaje. El análisis de regresión, que incorpora como predictores los resultados en los ejercitadores, muestra un fuerte impacto de este factor sobre el aprendizaje en las dos unidades. 
Para nuestra investigación, este efecto presenta valores más altos que los obtenidos en las variables experimentales de sincronía y heterogeneidad. Esta conclusión concuerda con los resultados de investigaciones previas, como la de Maldonado, Ortega, Ibáñez, Sanabria y Quintero (2003) en la cual se utiliza un agente de software, denominado Agente Q, como mecanismo para que el estudiante organice estructuras de conceptos en forma de categorías ontológicas relacionadas. Los resultados obtenidos con este Agente durante las sesiones de estudio se correlacionan positivamente con los resultados de evaluación del aprendizaje obtenido al final de la unidad. En una segunda investigación realizada por Maldonado, Ortega, Rodríguez y Macías (2003) se incorpora un agente generador de preguntas en un hipertexto, la solución a los problemas presentados por el agente es predictor del éxito en una evaluación sobre el aprendizaje del contenido del hipertexto.

\section{Conclusiones}

Los resultados obtenidos en esta investigación, a partir de los datos estadísticos, son argumentos suficientemente sólidos para sustentar las conclusiones que se presentan a continuación:

1. El aprendizaje de los estudiantes es afectado por la dinámica de trabajo, y se manifiesta en un rendimiento superior de los grupos heterogéneos frente a los grupos homogéneos.

Esta conclusión motiva a considerar el efecto de la colaboración entre estudiantes en los escenarios virtuales de aprendizaje, que es sin duda una dimensión fundamental si se desea desarrollar una pedagogía científicamente validada para garantizar calidad de la oferta educativa, como es el caso de la educación a distancia en la actualidad Dado que ésta es una conclusión global, una investigación que se concentre en este aspecto podría brindar mas información sobre las características específicas de la colaboración entre los actores del aprendizaje en aulas virtuales.

2. El modelo de reducción de diferencias, específicamente los mecanismos de monitoreo y regulación, juega un papel decisivo en la calidad del aprendizaje en aulas virtuales.

Si bien esta investigación se orientó por el uso de un modelo integrador de factores MRD, no tuvo como propósito estudiar este modelo desde un punto de vista dinámico, sin embargo, en la actualidad se dispone de un escenario que permite hacer tipo de estudio, investigando el peso relativo de cada componente del modelo y las relaciones entre dichos dispositivos. Por ejemplo, el uso de estrategias de resolución de problemas que se aprende con la práctica tiene efecto en la precisión de los juicios de metamemoria, en el aprendizaje de solución de problemas y en el significado del trabajo colaborativo. Esta investigación está mostrando que el MRD puede jugar un papel clave en el mejoramiento de la calidad del aprendizaje en aula virtual y en escenarios no virtuales.

3. Se pueden considerar dos explicaciones para el incremento del nivel de aprendizaje de la primera a la segunda unidad, primero, la adaptación al entorno, que daría un argumento en favor del Modelo de Reducción de Diferencias y segundo, el hecho de haber conseguido dominar un conocimiento, el de la primera unidad, permite que el trabajo realizado comience a interactuar con otras formas 
de conocimiento, como las que aparecen en la segunda unidad.

4. No se muestran diferencias relacionadas con el uso del chat y del foro.

Esta conclusión amerita un análisis más profundo. En efecto, en esta investigación no se hizo un entrenamiento específico a los tutores siguiendo un modelo particular, es probable que si se introducen modelos de interacción, por parte de los tutores en cada uno de los escenarios virtuales, los resultados puedan cambiar. Como con la introducción de modelos que permitan la formulación de preguntas, ya que ésta es una habilidad que a primera vista necesita desarrollarse; o con la posibilidad de cambiar la dirección de las preguntas para que se orienten de mejor manera los procesos de aprendizaje; también

\section{Referencias bibliográficas}

Bodzin, A. (2000). Non-restricted asynchronous web-based forums: a study of preservice science teachers' attitudes.International Journal of Educational Telecommunications, 6(4), 363-392.

Castañeda, A. y Jaime, D. (2006).Interacción de docentes universitarios en un foro virtual en un proceso de educación no formal. Bogotá: Universidad de La Salle.

Ddillenborug, P.; Mendelsohn, P. y Scheneider, D. (1994). The distribution of pedagogical roles in a multi-agent learning environment. En R. Lewis y P. Mendelsohn (eds.), Lessons from Learning. (pp.199-216). North-Holland.

Dunlosky, \& J. Hertzog, C. (1998). Training programs to improve learning in later adulthood: helping older adults educate themselves. In Haec- si se da la posibilidad de manipular la información que retorna; o permitir el manejo de la información y el uso de referentes textuales y contextuales. El estudio de estos mecanismos requiere de acercamientos tanto analíticos como holísticos para una mejor comprensión de este componente. La investigación que se presenta en este artículo logró desarrollar un análisis de interacción presentado en Maldonado y otros (2007)

Finalmente, los resultados que se presentan en esta investigación corresponden a una población constituida por estudiantes de primeros semestres de pregrado, aún no se han consolidado resultados con una población estudiantil de otros niveles educativos.

ker, D. J., Dunlosky, J. y Graesser A. C. (eds.),Metacognition in educational theory and practice. Mahwah, $\mathrm{NJ}$ : Lawrence Erlbaum Associates.

Facundo, D. y Ánge, H. (2003). La educación superior virtual en Colombia. UNESCO, 26.

Harpe, B.; Mackensie, A. y Technikon, C. (2002). Chat rooms as an academic teaching technique. Extraído de http://citte.nu.ac.za/papers/id2.pdf.

Maldonado, L.; Ortega, N.; Sanabria, L. y Mora, D. (2003). Question Generator Software Agents versus shells for building hypertext: effects on conceptual structure comprehension. Capítulo 23. En M. Llamas, M. J. Fernández y L. E. Anido (Edits.),Computers and Education: Towards a Lifelong Learning Society. Netherlands: Kluwer Academic Publishers (pp.275-284). España. 
Maldonado, L.; Ortega, N.; Ibañez, J.; Sanabria, L. y Quintero, V. (2003). Agente de software Q y el aprendizaje de modelos conceptuales. Tecné, Episteme y Didaxis, 14. 95-112.

Maldonado, L. (2004). Competencias docentes en el contexto de los ambientes virtuales de aprendizaje. Encuentro Iberoamericano de Formación Docente "Entre orugas y mariposas" (pp. 135-154). Vol. 1. Bogotá: Universidad Pedagógica Nacional.

Maldonado, L. (2005) (en prensa).Virtualidad y autonomía: pedagogía para la equidad. Bogotá: Universidad Pedagógica Nacional.

Maldonado, L., Leal, L., Montenegro, M., Pérez, J. y Lineros, C. (2005). Solución de Problemas y consolidación de grupos: Incidencia del número de participantes en un ambiente de chat. Informe de Investigación. Bogotá: Universidad Nacional Abierta y a Distancia.
Singh, V.; Khassawneh, M.; Bowling, S.; Jiang, X.; Master, R. y Gramopadhye, A. (2002). La evaluación de sistemas alternativos de aprendizaje: Asincrónico, sincrónico y salón de clase. Department of Industrial Engineering Clemson, University Clemson, SC29634-0920.Extraido de http://www.ws.binghamton. edu/ mkhasawn/Publications/Conference/ Other/ICCAES\%202002\%20(Learning\%20Systems).pdf.

Sowa, J. F. (2000). Knowledge Representation: logical, philosophical, and computacional foundations. Boston, MA: Brooks/Cole, Thomson Learning.

Thiede, K.; Anderson, M. y Therriault, D. (2003). Accuracy of metacognitive monitoring affects learning of texts. Journal of Educational Psychology, 93, 1, 66-73. 